The Astrophysical Journal, 299:L1-L5, 1985 December 1

(C) 1985. The American Astronomical Society. All rights reserved. Printed in U.S.A.

\title{
DISCOVERY OF A PROBABLE GALAXY WITH A REDSHIFT OF $3.218^{1}$
}

\author{
S. Djorgovski ${ }^{2,3}$, Hyron Spinrad ${ }^{2}$, Patrick McCarthy ${ }^{2}$, and Michael A. Strauss ${ }^{2,4}$ \\ Received 1985 August 1; accepted 1985 September 5
}

\begin{abstract}
We report the discovery of a narrow emission line object, probably a galaxy, with a redshift of 3.218 . The object is a companion to the quasar PKS $1614+051$, which is at a redshift of 3.209. This is the most distant non-QSO, non-gravitationally lensed object presently known by a large margin. Its properties are consistent with those expected of a high-redshift galaxy. This object has an age of only a few percent of the present age of the universe. The object was discovered with a novel technique, which promises to push studies of distant galaxies to redshifts as high as those of the most distant quasars known, and which may eventually lead to the discovery of primeval galaxies. This discovery opens the way for studies of galaxies beyond $z=3$, which should prove invaluable for observational cosmology.
\end{abstract}

Subject headings: cosmology - galaxies: evolution - galaxies: formation - galaxies: redshifts - quasars

\section{INTRODUCTION}

The study of galaxies over large look-back times is one of the main paths of observational cosmology. It is the most direct way of learning about the evolution of luminous matter in the universe, and it is a necessary step if one is to use galaxies as cosmological probes. The search for ever more distant galaxies has been one of the main tasks on the frontier of cosmological research, ever since the time of Hubble and Slipher. Inactive cluster galaxies are now known with redshifts up to 0.92 (Gunn 1982). The most distant (non-QSO) galaxies known until now were the powerful 3CR sources (Spinrad and Djorgovski 1984), with the record redshift of 1.819 for 3C 256. (We use the term "galaxy" to exclude QSOs, although we do believe that all QSOs are located in galaxies.) Many QSOs with higher redshifts are known, the most distant being PKS 2000-330, with $z=3.78$ (Peterson et al. 1982). Unfortunately, QSOs are poor cosmological (and galaxy evolution) probes, because of their extreme intrinsic variability, and because their light is mainly nonthermal, generated in a poorly understood central "engine." In this Letter, we report the discovery of a narrow emission line object with a redshift $z=3.218$, whose properties are approximately those which may be expected of a galaxy at such a high redshift.

This object is a companion of the QSO PKS 1614+051 (Shimmins, Bolton, and Wall 1975). We measure the QSO redshift to be $3.209 \pm 0.001$, whereas Jauncey et al. (1979), as quoted by Hewitt and Burbidge (1980), give $z_{\mathrm{QSO}}=3.208$. Its position was measured by Condon, Hicks, and Jauncey (1977), who give the finding chart, and estimate the QSO $V$ magnitude to be 19.5. An improved radio (VLBI) position of the

\footnotetext{
${ }^{1}$ Based in part on research done at Lick Observatory, University of California.

${ }^{2}$ Department of Astronomy, University of California, Berkeley.

${ }^{3}$ Harvard Junior Fellow.

${ }^{4}$ Department of Physics, University of California, Berkeley.
}

QSO is given by Morabito et al. (1982). PKS $1614+051$ is a compact radio source with an inverted spectrum (Wright 1983). The coincidence of the radio and optical positions is quite satisfactory. There is little doubt that the QSO, and not the companion, is the radio source.

The companion object was located with a new technique, which we describe below.

\section{THE OBSERVATIONS AND DATA REDUCTIONS}

Extremely distant galaxies are necessarily extremely faint, and devising methods to find them is an interesting problem in itself. For many years, the most distant galaxies known have been located by means of their powerful radio emission (often associated with optical line emission), and apparent optical faintness. Yet, in the spite of substantial effort, no galaxy has ever been found beyond $z=1.82$. One possible exception is the gravitationally lensed object $2016+112$ (Lawrence et al. 1984), with $z=3.273$.

If we can extrapolate from common experience with lowredshift galaxies, the best place to look for a galaxy is next to another galaxy, because of pervasive galaxy clustering. (The degree of clustering must evolve, however, and it may or may not be significant at $z>3$.) Thus, one can use the distant QSOs as "signposts" or "beacons," and look for their putative galaxy companions. $L_{*}$ galaxies (viz., galaxies of a "typical" luminosity) are expected from cosmological and evolutionary considerations (see, e.g., Djorgovski and Spinrad 1985a) to have visible magnitudes fainter than about 23 or 24 . This faintness precludes a continuum spectroscopy survey at the present time. Broad-band colors may be used as approximate redshift indicators (cf. Koo 1985a), but at very high redshifts $(z>3$, say), the uncertainties in our estimates of evolutionary effects are very large. This leaves only the narrow emission line galaxies as promising targets at very high redshifts, at least at the present time. One approach suggests itself: obtain 
direct images of high-redshift QSO fields in a very narrow band centered on a suitable emission feature (Ly $\alpha$ is the obvious choice), and also in a broad band that samples the nearby continuum. Any faint objects with a notable light "excess" in the Ly $\alpha$ band are then selected as possible emission-line galaxies at the target QSO redshift.

This is precisely what we have done. We used an interference filter centered at $\lambda=5139 \AA$, with a FWHM $=90 \AA$, corresponding to a Ly $\alpha$ redshift of 3.226. From the catalog of Véron-Cetty and Véron (1984) we then selected all QSOs in the appropriate redshift range observable from Lick in the 1985 July dark run. Out of five such QSOs, PKS $1614+051$ was judged to be the best initial choice. For the continuum comparison band, we used the $r_{S}$ band $\left(\lambda_{\text {eff }}=6890 \AA\right)$, which is defined by Djorgovski (1985).

All observations were done with the Cassegrain CCD system (Miller 1983; Lauer et al. 1983) on the Lick Observatory $3 \mathrm{~m}$ Shane telescope. The discovery images were obtained on the night of 1985 July $18 \mathrm{UT}$, in good seeing conditions $(F W H M \approx 1$ 1.5). Two Ly $\alpha$ images with exposure times of $1200 \mathrm{~s}$ each and two $r_{s}$ images with exposure times of $300 \mathrm{~s}$ each were obtained; all images were taken in the position angle (PA) of $90^{\circ}$. The telescope was offset a few arcsec between adjacent exposures; this practice assures the repeatability of detected objects as they are imaged with different portions of the $\mathrm{CCD}$ each time, and it also improves the flat-fielding. It was immediately apparent that there was a strong Ly $\alpha$ “excess" object next to the QSO. Three additional direct $r_{S}$ images with exposure times of $200 \mathrm{~s}$ each were obtained on the night of 1985 July 19 UT, in equally good seeing conditions, and PA $=43^{\circ}$. Three more direct $r_{S}$ images with exposure times of $207 \mathrm{~s}$ each were obtained on the night of 1985 August $15 \mathrm{UT}$, and two images of $207 \mathrm{~s}$ and one of $345 \mathrm{~s}$, on the night of 1985 August $16 \mathrm{UT}$, all in FWHM $\approx 1 . .2$ seeing conditions, and with $\mathrm{PA}=201^{\circ}$. (The odd PAs were dictated by the spectroscopy.)

The flat-fielding of the broad-band images was accomplished using a sky median frame for the appropriate night (Djorgovski 1984). The narrow-band images were flat-fielded with exposures of the twilight sky, a permissible practice for narrow-band CCD imaging. The images were interpolated to twice the original sampling (to a new pixel size of 0 ".365) by bilinear interpolation, digitally rotated if necessary, and then registered and stacked together. Images of the flux standard Feige 98 (Stone 1977) were used both for the monochromatic ( $\lambda$ 5139) and $r_{S}$ magnitude calibrations. Digital stacks of the appropriately rotated and shifted images are shown in Figure 1 (Plates L1 and L2).

Spectroscopic observations were initially obtained on the night of 1985 July 19 UT. The seeing FWHM was about $1^{\prime \prime}-1{ }^{\prime \prime} .5$ at the start but deteriorated somewhat as the objects began to set. The transparency also gradually deteriorated, because of a variable high-altitude smoke aerosol. The Cassegrain spectrograph slit was rotated to $\mathrm{PA}=43^{\circ}$, to cover both the QSO and the companion. One $3600 \mathrm{~s}$ spectroscopic exposure with a low-resolution (420 grooves $\left.\mathrm{mm}^{-1}\right)$ grism,

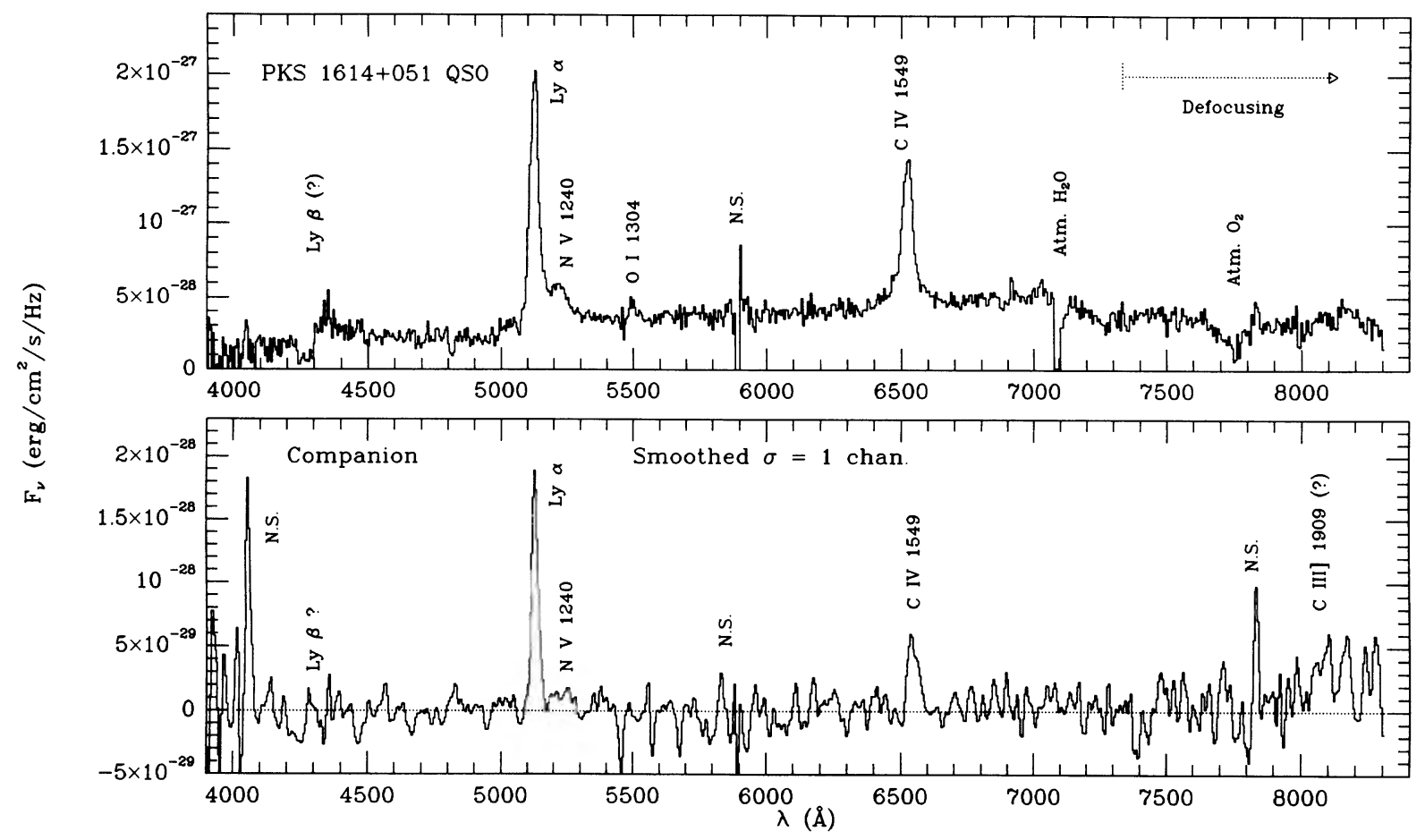

FIG. 2. - Spectra of the PKS 1614+051 QSO (top) and its companion object (bottom). The QSO spectra are from the July data only, and the companion spectra are a quality-weighted average of July and August data. The companion object spectrum was smoothed with a narrow Gaussian filter in order to supress the pixel-to-pixel noise; this smoothing also causes some artificial broadening of the emission lines. Dubious line identifications are indicated with a question mark (?). Spurious features caused by imperfect night sky subtraction are indicated (N.S.). CCD defocusing in the red causes spurious dimming of the QSO continuum, some of which spills over to the companion object's spectrum, and biases the companion continuum. 


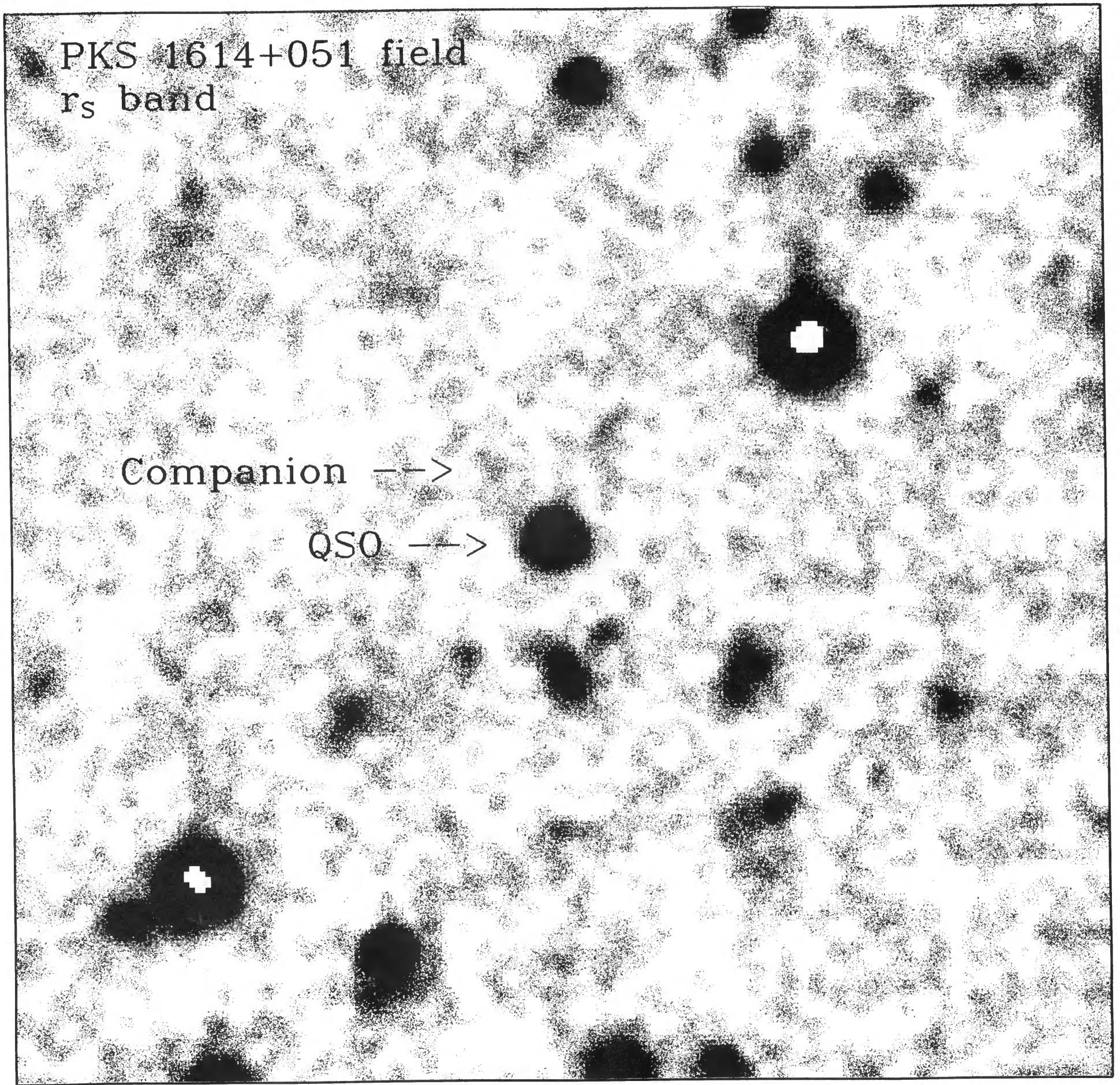

FIG. $1 a$

FIG. 1.-Stacked CCD images of the PKS 1614+051 QSO field. North is at the top, east to the left, and the fields are 73 arcsec ${ }^{2}$. The QSO coordinates are: $\alpha=16^{\mathrm{h}} 14^{\mathrm{m}} 09^{\mathrm{s}} .1, \delta=05^{\circ} 06^{\prime} 54^{\prime \prime}(1950)$ : ( $a$ ) Broad-band red image; the companion is barely detected. Many faint, red (almost certainly foreground) galaxies are present. The data are from both July and August runs. (b) Ly $\alpha$ image; note the prominence of the companion object. The data are from the July run only.

DJORGovsKI et al. (see page L2) 


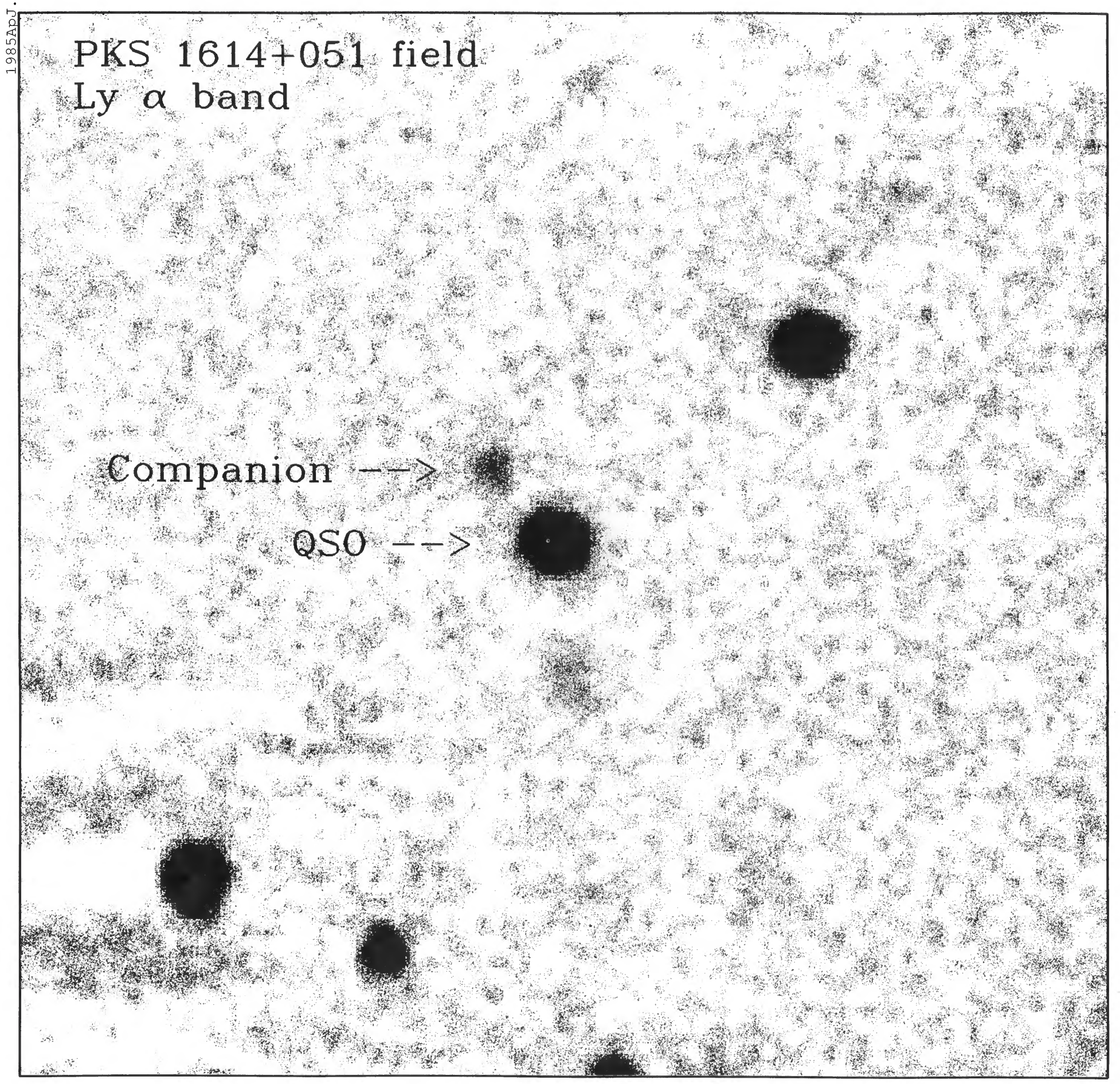

FIG. $1 b$

DJORGOvSKI et al. (see page L2) 
and one $3600 \mathrm{~s}$ exposure with a medium-resolution $(600$ grooves $\mathrm{mm}^{-1}$ ) grism were obtained. In this way the spectroscopic features were sampled with different dispersers and different positions on the CCD. The wavelength calibrations have rms errors of about $5 \AA$ and $1 \AA$, respectively; we used the high-resolution data to measure the redshifts. The spectral resolutions of the two grisms are FWHM $\approx 15 \AA$ and $10 \AA$, respectively. Additional spectroscopic exposures were obtained on the nights of 1985 August 15 UT (2480 s integration), and 1985 August 16 UT (2760 s integration). Both of these exposures used the " 420 " grism, and the slit PA $=201^{\circ}$, centered on the companion object and the red galaxy just south from the QSO (see Fig. 1). The seeing was excellent, with $\mathrm{FWHM} \approx 1^{\prime \prime} 2$.

The spectra were reduced with the methods described in Djorgovski and Spinrad (1983), and flux-calibrated with the exposures of Feige 98 (Stone 1977). The averaged spectra are shown in Figure 2. The reality of the weak emission-line features in the spectrum of the companion was verified by direct visual examination of the two-dimensional, sky-subtracted spectroscopic frames.

\section{THE RESULTS}

From our direct images, we obtain magnitudes for the QSO and the companion:

$$
\begin{gathered}
r_{S, \mathrm{QSO}}=19.69 \pm 0.01, \quad m(5139)_{\mathrm{QSO}}=17.09 \pm 0.01, \\
r_{S, \mathrm{COMP}}=24.56 \pm 0.5, \quad m(5139)_{\mathrm{COMP}}=20.12 \pm 0.02,
\end{gathered}
$$

where $\mathrm{m}(5139)$ pertains to the whole FWHM $=90 \AA$ band, and the positional offsets:

$$
\begin{gathered}
\Delta \alpha_{\text {COMP-QSO }}=+4^{\prime \prime} .20 \pm 0^{\prime \prime} .05, \\
\Delta \delta_{\text {(COMP-QSO) }}=+5^{\prime \prime} .04+0^{\prime \prime} .05,
\end{gathered}
$$

corresponding to a separation of 6".55 and a position angle of $40^{\circ}$. Taking the QSO position from Morabito et al. (1982), we obtain the position of the companion object (1950 epoch):

$$
\alpha=16^{\mathrm{h}} 14^{\mathrm{m}} 09^{\mathrm{s}} 37, \quad \delta=+05^{\circ} 06^{\prime} 58^{\prime \prime} .9,
$$

with estimated total errors of about 0.77 in each coordinate, which includes our $00^{\prime \prime} 05$ offset errors, and 0".6-0".7 positional errors from Morabito et al.

Surface brightness profiles for the QSO and the companion object are shown in Figure 3. Apparently, the companion is unresolved in both bands, although the signal is poor in the $r_{S}$ band.

The measured redshift for the companion object is $z_{\text {COMP }}$ $=3.2182 \pm 0.0005$, based on the Ly $\alpha$ line. The peak of the $\mathrm{C}$ IV line gives $z_{\text {COMP }}=3.219$, in good agreement with the Ly $\alpha$ redshift, but this line is broadened in its red wing, probably due to a noise spike. This agreement of redshifts suggests that the Ly $\alpha$ line is not significantly self-absorbed in its blue wing, as is the case with the QSO. The other lines, as indicated in Figure 2, are too weak for reliable measurement. The measured equivalent width of the Ly $\alpha$ line in the compa-

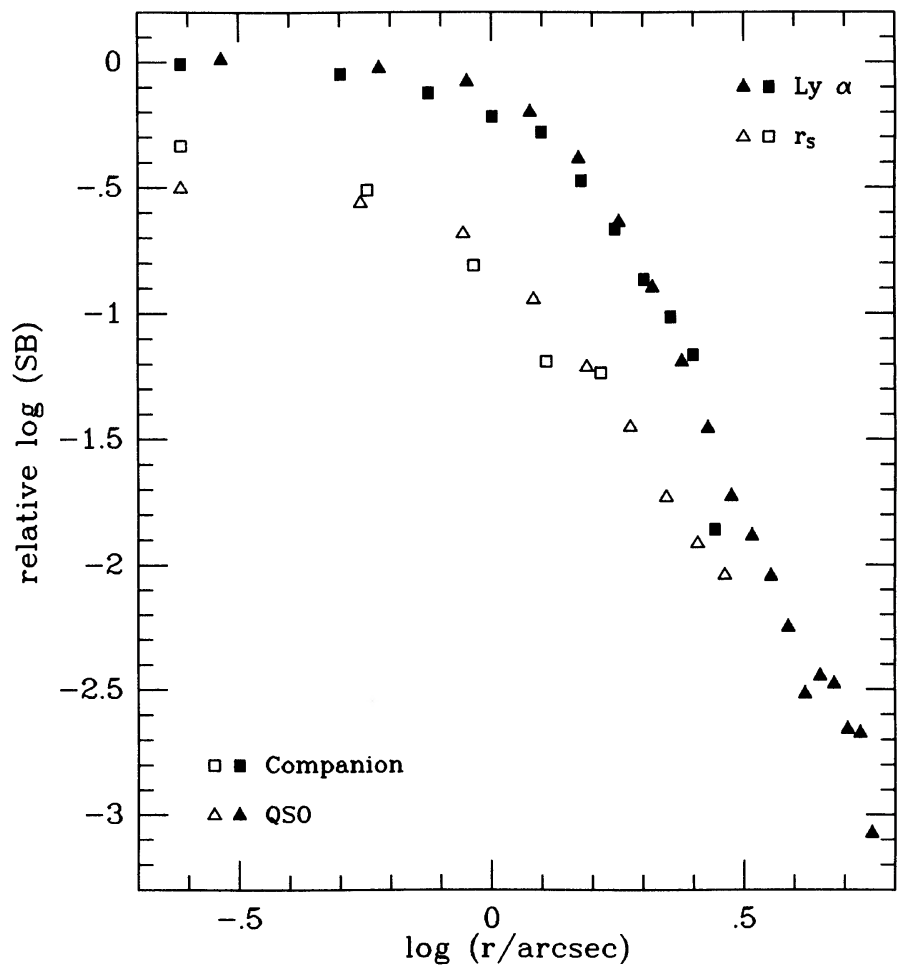

FIG. 3.-Surface brightness profiles for the QSO (triangles) and the companion object (squares), obtained in the redshifted Ly $\alpha$ band (solid symbols) and the broad-band red continuum (open symbols). The curves were shifted along the $Y$-axis so that the surface brightnesses for the two objects roughly match in each band. Red profiles where shifted down by 0.5 dex, for clarity. Ly $\alpha$ band data are from the July run only, and the red data are from both July and August runs.

nion object is about $200 \AA$ in the rest frame, similar to that found in high-redshift radio galaxies (Spinrad et al. 1985). The continuum is very weak, and this number should perhaps be regarded as a lower limit. Its intrinsic width (corrected for the instrumental resolution) is about $900 \pm 300 \mathrm{~km} \mathrm{~s}^{-1}$ FWHM in the rest frame, which is appropriate for a mildly active galaxy. The overall spectrum appearance is very similar to that of high-redshift radio galaxies (Spinrad and Djorgovski 1984; Spinrad et al. 1985). The poor signal-to-noise ratio and troublesome sky subtraction should not be too surprising: at $24 \mathrm{~m} .5$, this is probably the faintest object for which a conclusive spectrum has ever been obtained.

The QSO has a normal, high-redshift QSO spectrum. We measure its redshift from the $\mathrm{Ly} \alpha$ line to be $z_{\mathrm{QSO}}=3.214$. The Ly $\alpha$ line may be self-absorbed in its blue wing and has perturbing N v 1240 emission in its red wing. From the C IV 1549 line, we find a redshift $z_{\mathrm{QSO}}=3.209$. Thus, the companion object is redshifted with respect to the QSO by some 300 $\mathrm{km} \mathrm{s}^{-1}$ in the rest frame if the QSO Ly $\alpha$ redshift is used, or about $660 \mathrm{~km} \mathrm{~s}^{-1}$ if the C IV line QSO redshift is used, with errors of about $90 \mathrm{~km} \mathrm{~s}^{-1}$.

Note that the line ratios and equivalent widths are quite different in the QSO and the companion object: the Ly $\alpha$ to $\mathrm{C}$ Iv line energy ratios are 1.96 and 3.5 for the QSO and the companion, respectively, and the QSO lines have the rest 
frame FWHM of $\sim 2000 \mathrm{~km} \mathrm{~s}^{-1}$ The redshift difference (which is also very apparent in the spectroscopic CCD frames, at least for Ly $\alpha$ ), along with the differences in line widths and ratios, rules out the possibility that we are seeing yet another example of gravitational lensing.

Our spectra of the red galaxy just south from the QSO are still too poor to yield a redshift; no emission lines are present. The galaxy is almost certainly a foreground object. The number of faint galaxies in our red CCD frames suggests a possible foreground cluster.

\section{DISCUSSION AND CONCLUSIONS}

The companion object is certainly young, because of its high redshift. In a $q_{0}=0 \quad \Lambda_{0}=0$ Friedman-Lemaitre cosmology, the look-back time to this system is 0.763 of the age of the universe ( $=$ the Hubble time), whereas in a $q_{0}=1 / 2$ $\Lambda_{0}=0$ cosmology, it is 0.855 of the age of the universe (=2/3 of the Hubble time). If the galaxies formed at $z=5$ (which currently seems to be a reasonable guess), we are seeing this system at an age of only $8.4 \%$ or $5.0 \%$ of the present galaxian age, for the two cosmological models above. If galaxies formed at $z=10$, these numbers become $16.1 \%$ and $9.1 \%$, respectively. The corresponding numbers for PKS $2000-330$ (the most distant QSO known, at $z=3.78$ ) differ from these by only a couple of percent.

What is the nature of the companion object? Its spectrum is definitely not that of the QSO/Seyfert 1 type, and it is not very different from those of radio galaxies with $z \approx 1.5-2$. The Ly $\alpha$ line to local continuum ratio is very high, in contrast to ratios seen in the QSO/Seyfert 1 spectra. The fact that it is unresolved in our direct images should not be surprising: at such a high redshift, only the central parts of galaxies should be observable, since relativistic $(1+z)^{4}$ surface brightness dimming supresses their faint envelopes, even though stellar population evolution would make the envelopes brighter (by a poorly known amount). One possibility is that we are seeing an equivalent of the nuclear starburst disk of NGC 1068 (Weedman and Huenemoerder 1985). We may have to wait for the Hubble Space Telescope in order to resolve the morphology of this object. It is certainly possible that this object is a marginal Seyfert 2, or a LINER. It does possess a faint red continuum, which may well be due to starlight. Note the presence of carbon and possibly nitrogen lines in its spectrum - some reprocessing of gas has already happened.

Djorgovski and Spinrad (1985a) have found that the colors and magnitudes of $3 \mathrm{CR}$ galaxies (presumably giant ellipticals) at high redshifts are described well by the continuous, exponentially decaying star formation models of Bruzual A. (1981, 1983). The "best" models (Bruzual $\mu=0.5-0.7)$ predict red magnitudes of the order of $22-23$ for these very luminous galaxies at $z \geq 3$. If such models can indeed be extrapolated and applied in this case (a very tenuous assumption), we may be seeing an actively evolving $L_{*}$ galaxy; if milder evolution is assumed, the galaxy should be of a brighter kind. The conclusion is that nothing more dramatic than a moderately luminous galaxy (e.g., like our Galaxy) is required. The distance moduli $(m-M)$ at this redshift, for the two cosmological models mentioned above, are 46.99 and 45.56, respectively, for $H_{0}=100 \mathrm{~km} \mathrm{~s}^{-1} \mathrm{Mpc}^{-1}$; one must still subtract the $K$-corrections and evolutionary effects from these numbers.

The projected separation between the QSO and the companion corresponds to 45 or $23 h_{100}^{-1} \mathrm{kpc}$ for the two cosmologies above. Their relative velocity is typical of that observed in moderately rich clusters or rich galaxy groups at low redshifts. Two objects do not a cluster make, but the possibility of clustering at such high redshifts is very intriguing-it is a fundamental test for the theories of large-scale structure and galaxy formation.

Another possibility is that the companion is a gas-rich galaxy, with the narrow-line emission excited by the QSO's radiation field. This is very seldom the case for QSO companion galaxies at low redshifts (Filippenko 1985; Heckman et al. 1984; Djorgovski and Spinrad 1985b). If more such galaxy companions to high-redshift QSOs are found in the future, we may be able to address this possibility. Another possibility is that we are seeing a high-redshift equivalent of Minkowski's object (van Breugel et al. 1985; Brodie, Bowyer, and McCarthy 1985), a gas-rich galaxy excited by a radio jet. A modern VLA map could check the latter possibility. Measurements of its near-infrared continuum would also be very valuable, in order to constrain its stellar content.

The prominent Ly $\alpha$ emission in this object (and the equally prominent emission found in $z \approx 1.5-2$ radio galaxies by Spinrad et al. 1985) gives new hope for primeval galaxy searches. The pessimistic predictions of Hartman, Huchra, and Geller (1984) about Ly $\alpha$ quenching are based on lowredshift, gas-rich dwarfs; these authors may still be right about the spectroscopic dullness of some or even most primeval galaxies, but there are clearly galaxies at high redshifts with very prominent Ly $\alpha$ emission. The models of Meier (1976) and Cox (1985) predict an early Ly $\alpha$-luminous phase of primeval galaxies. The properties of primeval galaxies predicted by Meier (1976), viz., compact appearance and strong Ly $\alpha$, agree well with our observations of the companion object. (See also the reviews by Davis 1980 and Koo 1985b on how to recognize a primeval galaxy.) It now appears that the fall-off in the number density of QSOs beyond $z=3$ is real (Schneider, Schmidt, and Gunn 1984). If the appearance of QSOs reflects the appearance of galaxies, i.e., if galaxies formed at $z \leq 4$, then we may have discovered a good approximation to a primeval galaxy.

Perhaps the most important result of the present work is the proof of the method: it is possible to find extremely distant, young galaxies, using interference filter imaging of highredshift QSO fields (and perhaps even “blank” fields). Unless we are victims of improbable good luck, we should be able to find more such distant objects. If so, we will have succeeded in pushing the studies of distant galaxies well into the "realm of the quasars." The next step, of course, is a search for (primeval?) galaxies at $z=4$, and beyond. One such experiment is now being carried out by C. Pritchet and D. Hartwick (C. Pritchet, private communication).

We are grateful to the staff of Lick Observatory for their constant and untiring help and assistance, and in particular to B. Alcott, K. Baker, W. Earthman, and R. Stone. The Shane telescope CCD system is being developed by J. Miller, L. 
Robinson, R. Stover, and their collaborators. The magnitudes of the QSO and the companion objects were measured with the program DAOPHOT, developed by P. Stetson. This work was partly supported by the NSF grant AST85-42631 and the NASA grant NAGW-270 to H. S. M. A. S. acknowledges the support of an NSF Graduate Fellowship.

\section{REFERENCES}

Brodie, J. P., Bowyer, S., and McCarthy, P. 1985, Ap. J. (Letters), 293, L59.

Bruzual A., 1981, Ph. D. thesis, University of California, Berkeley. 1983, Ap. J., 273, 105 .

Condon, J. J., Hicks, P. D., and Jauncey, D. L. 1977, A.J., 82, 692.

Cox, D. P. 1985, Ap. J., 288, 465.

Davis, M. 1980, in IAU Symposium 92, Object of High Redshift, ed. G. O. Abell and P. J. E. Peebles (Dordrecht: Reidel), p. 57.

Djorgovski, S. 1984, in Proc. of Workshop on Improvements to Photometry, ed. W. Borucki and A. Young (NASA CP-2350), p. 152. 1985, Pub. A.S.P., in press.

Djorgovski, S., and Spinrad, H. 1983, in Proceedings of the AAS/OSA Joint Topical Meeting on Information Processing in Astronomy and Optics (Washington: Optical Society of America), p. ThB2-1. .1985a, Ap. J., submitted. 1985 b, Bull. AAS 17,516

Filippenko, A. V. 1985, A.J., 90, 1172.

Gunn, J. E. 1982, in Astrophysical Cosmology, ed. H. A. Brück, G. V. Coyne, and M. S. Longair, Pontif. Acad. Sci. Scripta Varia, 48, 233.

Hartman, L. W., Huchra, J. P., and Geller, M. J. 1984, Ap. J., 287, 487.

Heckman, T., Bothun, G. D., Balick B., and Smith, E. P. 1984, A.J., 89, 958.

Hewitt, A., and Burbidge, G. 1980, Ap. J. Suppl., 43, 57.

Jauncey, D. L., Wright, A. E., Peterson, B. A., and Condon, J. J. 1979, unpublished (?) preprint, cited in Hewitt and Burbidge 1980.

Koo, D. C. $1985 a$, A.J., $90,418$.

Koo, D. C. $1985 b$, preprint

Lauer, T. R., Miller, J. S., Osborne, C. S., Robinson, L. B., and Stover, R. J. 1983, Proc. Soc. Photo-Opt. Instr. Eng., 445, 132.

Lawrence, C. R., Schneider, D. P., Schmidt, M., Bennet, C. L., Hewitt, J. N., Burke, B. F., Turner, E. L., and Gunn, J. E. 1984, Science, 223, 46.

Meier, D. L. 1976, Ap. J., 207, 343.

Miller, J. S. 1983, Lick Observatory internal report.

Morabito, D. D., Preston, R. A., Slade, M. A., and Jauncey, D. L. 1982, A.J., 87, 517.

Peterson, B. A., Savage, A., Jauncey, D. L., and Wright, A. E. 1982, Ap. J. ( Letters), 260, L27.

Schneider, D., Schmidt, M., and Gunn, J. 1984, Bull. AAS, 16, 488.

Shimmins, A. J., Bolton, J. G., and Wall, J. V. 1975, A ustralian J. Phys. Suppl., 34, 63.

Spinrad, H., and Djorgovski, S. 1984, Ap. J. (Letters), 285, L49.

Spinrad, H., Filippenko, A. V., Wyckoff, S., Stocke, J. T., Wagner, M., and Lawrie, D. G. 1985, Ap. J. (Letters), 299, L00.

Stone, R. P. 1977, Ap. J., 218, 767.

van Breugel, W., Filippenko, A. V., Heckman, T., and Miley, G. 1985, Ap. J., 293, 83 .

Véron-Cetty, M.-P., and Véron, P. 1984, ESO Scientific Report Number 1. Weedman, D. W., and Huenemoerder, D. P. 1985, Ap. J., $291,72$.

Wright, A. E. 1983, in Quasars and Gravitational Lenses, Proc. 24th Liège Ap. Coll., (Liège: Institut d'Astrophysique), p. 50.

S. DJoRgovski: Center for Astrophysics, 60 Garden Street, Cambridge, MA 02138

P. MCCarthy, H. SpInrad, and M. A. Strauss: Astronomy Department, University of California, Berkeley, CA 94720 